\title{
Fußball senkt den Blutdruck hypertoner Frauen
}

\section{Bisher stand Fußball eher im Verdacht, den Blutdruck nach oben zu treiben. Bei Frauen hat die Kickerei offenbar den gegenteiligen Effekt - aber nur, wenn sie selbst gegen den Ball treten.}

19 Däninnen um die 40 mit leichter Hypertonie und einem Body-Mass-Index von mehr als 25 hatten zwei- bis dreimal wöchentlich an einem einstündigen Fußballtraining teilgenommen.

Nach einem Jahr war der mittlere arterielle Blutdruck der Fußballerinnen im Schnitt um $5 \mathrm{mmHg}$ gesunken. In einer Kontrollgruppe von 12 nicht trainie- renden Frauen war er dagegen um 4 mmHg gestiegen. Das Körpergewicht hatte um 2,5 kg abgenommen (Kontrollen: Zunahme um 0,6 kg), die Körperfettmasse um 3,2 kg (+0,2 kg). Knochenmineraldichte und Mineralgehalt der Knochen hatten bei den Fußballerinnen ebenfalls zugelegt. Auch Lipidprofil, körperliche Ausdauer und Leistungsfähigkeit waren durch das Training günstig beeinflusst worden.

Studienleiter Prof. Peter Krustrup, Sport- und Gesundheitswissenschaftler an der Universität in Odense, hat übrigens nicht nur hypertone Frauen im

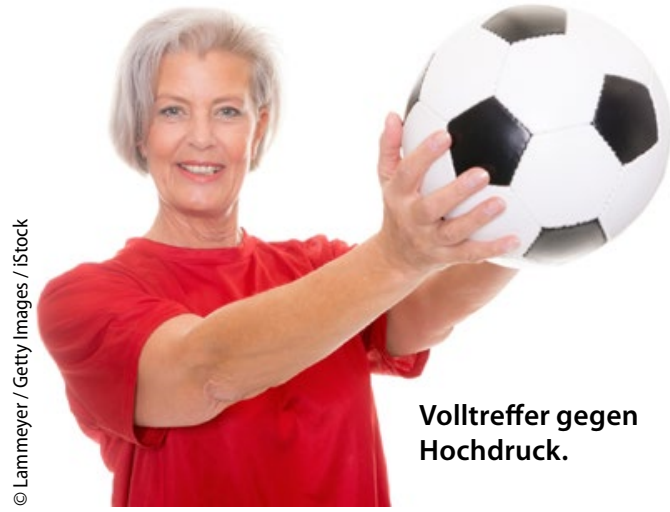

Blick. Zu seinen Konzepten gehört auch der FC Prostata, für den Männer mit Prostatakrebs aufs Feld laufen. Fußballerisch versuchen sie, gegen die Nebenwirkungen der Androgendeprivation anzuspielen sowie zu Teamgeist und Lebensmut zu finden. Das Projekt ist im vergangenen Jahr mit dem Dänischen Sportpreis ausgezeichnet worden. - rb

- Krustrup P et al. Scand J Med Sci Sports, online Januar 2017; doi: 10.1111/sms.12829

\section{Mit „Geistesnahrung“ gegen den kognitiven Abbau?}

\section{Lässt sich mit geistiger Tätigkeit dem kognitiven Abbau im Alter entgegen- wirken? Hinweise darauf haben US-For- scher in einer großen Kohortenstudie gefunden.}

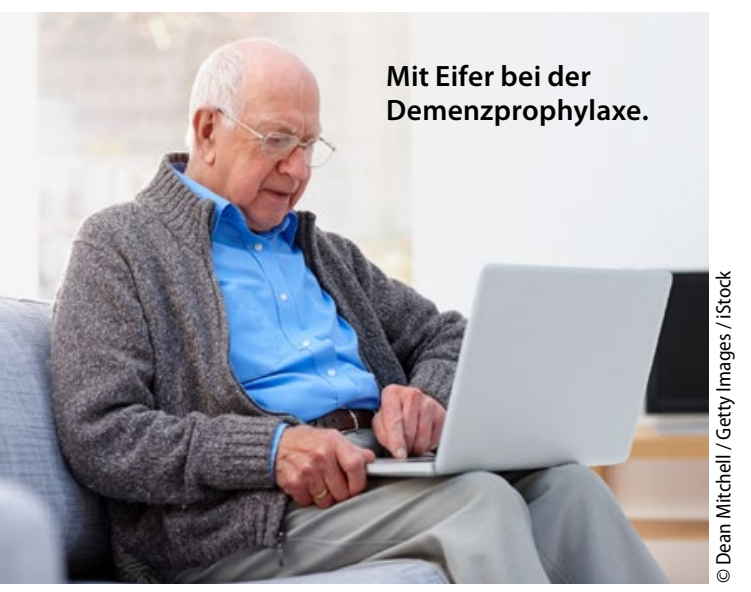

_ Die Studie greift auf Daten der Mayo Clinic Study of Aging (MCSA) zurück. 2.213 Senioren, die zu Beginn kognitiv unbeeinträchtigt waren, wurden im Mittel vier Jahre lang beobachtet und im Abstand von jeweils 15 Monaten mittels Fragebogen und kognitiver Tests untersucht. Gefragt wurde u.a., wie oft die Teilnehmer im Vorjahr ein Buch gelesen, sich mit Handarbeiten beschäftigt, ins Kino oder Theater gegangen waren, am Computer gesessen oder Gesellschaftsspiele gespielt hatten.

456 der 1.929 Teilnehmer hatten im Studienverlauf eine leichte kognitive Störung (MCI) entwickelt. Unter Berücksichtigung potenzieller Einflussfaktoren wie Alter, Geschlecht und Bildungsgrad errechneten die Forscher folgende Risikoreduktionen: Wer häufig, d.h. mindestens ein- bis zweimal pro Woche Spiele spielte, konnte sein MCIRisiko um 22\% senken, bei gesellschaftlich aktiven Teilnehmern betrug die Reduktion 23\%, bei handwerklich Tätigen 28\%. Als Referenzgruppen galten Personen, die die jeweiligen Aktivitäten höchstens zwei- bis dreimal im Monat aufgenommen hatten. Am stärksten war die Assoziation mit der „Arbeit“ am Computer: Hier betrug die Risikoreduktion 30\%. Bücherlesen ging nur mit einer (nicht signifikanten) Reduktion des MCI-Risikos um 17\% einher.

Neben reger Geistestätigkeit empfehlen die Forscher körperliche Fitness und eine kalorienbewusste Ernährung, um im Alter geistig fit zu bleiben. = eo

- Krell-Roesch J et al. JAMA Neurol 2017, online 30. Januar; doi: 10.1001/jamaneurol.2016.3822 